Ronald E. Jung

Vladimir Kokovic

Milan Jurisic

Duygu Yaman

Karthikeyan Subramani

Franz E. Weber

\title{
Guided bone regeneration with a synthetic biodegradable membrane: a comparative study in dogs
}

Key words: dog study, guided bone regeneration, PLGA membrane

Authors' affiliations:

Ronald E. Jung, Karthikeyan Subramani, Department of Fixed and Removable Prosthodontics and Dental

Material Science, Dental School, University of Zurich,

Zurich, Switzerland

Vladimir Kokovic, Milan Jurisic, Clinic of Oral

Surgery, Faculty of Dentistry, University of Belgrade, Belgrade, Serbia

Duygu Yaman, School of Dentistry, Department of Periodontology, Istanbul University, Istanbul, Turkey Franz E. Weber, Oral Biotechnology \& Bioengineering, Department of Cranio-Maxillofacial Surgery,

University Hospital, Zurich, Switzerland

\section{Corresponding author:}

Ronald E. Jung

Department of Fixed and Removable Prosthodontics and Dental Material Science

Dental School

University of Zurich

Plattenstrasse II

$\mathrm{CH}-8032$ Zurich

Switzerland

Tel.: + 4I 446343252

Fax: + 4I 4463443 O5

e-mail: ronald.jung@zzmk.uzh.ch
Date:

Accepted 8 August 2010

To cite this article:

Jung RE, Kokovic V, Jurisic M, Yaman D, Subramani K, Weber FE. Guided bone regeneration with a synthetic biodegradable membrane: a comparative study in dogs.

Clin. Oral Impl. Res. 22, 201 I ; 802-807.

doi: IO. I I I I/j. I600-050I .2010.02068.x

\section{Abstract}

Objectives: The aim of the present study was to compare a newly developed biodegradable polylactide/polyglycolide/N-methyl-2-pyrrolidone (PLGA/NMP) membrane with a standard resorbable collagen membrane (RCM) in combination with and without the use of a bone substitute material (deproteinized bovine bone mineral [DBBM]) looking at the proposed tenting effect and bone regeneration.

Materials and methods: In five adult German sheepdogs, the mandibular premolars P2, P3, P4, and the molar M1 were bilaterally extracted creating two bony defects on each site. A total of 20 dental implants were inserted and allocated to four different treatment modalities within each dog: PLGA/ NMP membrane only (Test 1), PLGA/NMP membrane with DBBM (Test 2), RCM only (negative control), and RCM with DBBM (positive control). A histomorphometric analysis was performed 12 weeks after implantation. For statistical analysis, a Friedman test and subsequently a Wilcoxon signed ranks test were applied.

Results: In four out of five PLGA/NMP membrane-treated defects, the membranes had broken into pieces without the support of DBBM. This led to a worse outcome than in the RCM group. In combination with DBBM, both membranes revealed similar amounts of area of bone regeneration and bone-to-implant contact without significant differences. On the level of the third implant thread, the PLGA/NMP membrane induced more horizontal bone formation beyond the graft than the RCM. Conclusion: The newly developed PLGA/NMP membrane performs equally well as the RCM when applied in combination with DBBM. Without bone substitute material, the PLGA/NMP membrane performed worse than the RCM in challenging defects, and therefore, a combination with a bone substitute material is recommended.

In the field of guided bone regeneration (GBR), there has been a dramatic reduction in the use of non-resorbable membrane like the expanded polytetrafluoroethylene (ePTFE), due to difficulties in handling, the need for a second surgery to remove the membrane, and the low reproducibility of results when membrane exposure had occurred resulting in wound infection and subsequently poor bone regeneration (Gher et al. I994; Simion et al. I994; Machtei 200I). To overcome these shortcomings, non-resorbable membranes were replaced in many indications by resorbable collagen membranes (RCMs) made from bovine or porcine sources (Parodi et al. I998; Hammerle \& Lang 200I). With improved biocompatibility, biodegradability, and better treatment results, most clinicians nowadays use RCMs (Lang et al. 2007; Hammerle et al. 2008). All collagen membranes originate from animal sources, which can cause difficulties for patient acceptance, immune responses, and a transmission of infectious agents can never be completely excluded. Few studies have reported that these membranes do exhibit unfavourable mechanical properties (Hurzeler et al. 1998) and inadequate barrier stability over time (Miller et al. 1996; Zhao et al. 2000; Owens \& Yukna 200I).

The usage of synthetic resorbable membranes made from aliphatic polyesters like polylactide, polyglycolide, and trimethylcarbonate has also been reported (Rosen \& Reynolds 200I; Kohal \& Hurzeler 2002). However, the degradation process of synthetic membranes made of polylactide or polyglycolide acids can impair bone regeneration due to adverse inflammatory tissue reaction (von Arx et al. 2002, 2005). Therefore, the development of a membrane based on biocompatible synthetic material with a long record 
of accomplishment in human use would represent an important step forward in GBR and in implantology. In this trial, a biodegradable fully synthetic membrane composed of polylactide, polyglycolide (PLGA), and trimethylene carbonate membrane was used as a barrier membrane for GBR in the dog mandible. The PLGA-based membrane is degradable by hydrolysis and releases $N$-methyl-2-pyrrolidone (NMP), which was shown to accelerate the maturation of preosteoblastic cells and bone regeneration in vitro and in vivo in a cranial defect model in rabbits that this PLGA/NMP membrane is capable of enhancing bone regeneration in a GBR model in humans as well. Unlike collagen-based membranes, PLGA-based membranes are stiff and can only be used clinically for GBR when softened by a plasticizer, such as NMP. Because NMP is released from the membrane when in contact with body fluids (Pirhonen et al. 2006), the applied membrane becomes stiff again, which might allow to omit the use of membrane-supporting materials. Owing to the low mechanical stability, collagen-derived membranes are mainly applied in combination with bone substitute materials (Jung et al. 2003; Cornelini et al. 2004).

The aim of this study was to compare the PLGA/NMP membrane with a standard RCM with and without the addition of deproteinized bovine bone mineral (DBBM) as bone substitute material and to determine their potential applications in GBR.

\section{Material and methods}

\section{Materials}

The collagen membrane Bio-Gide ${ }^{\mathbb{1}}$ and the bone substitute material Bio-Oss ${ }^{\circledR}$ were purchased from Geistlich AG (Wolhusen, Switzerland). The synthetic Inion ${ }^{\mathbb{R}} \mathrm{GTR}^{\mathrm{TM}}$ membrane and the resorbable pins were purchased from Inion $\mathrm{Oy}$ (Tampere, Finland). The Straumann Dental Implant System was purchased from Straumann AG (Basel, Switzerland). Both membranes were applied as recommended by the manufacturer. The following procedures was used for the novel PLGA/NMP membrane: After unwrapping, the stiff PLGA membrane was located in a tray. To soften the PLGA membrane, the supplied NMP solution was added to the tray and allowed to penetrate the membrane for $30 \mathrm{~s}$. After soaking, the membrane was taken from the tray with tweezers and placed for romin in the second part of the tray to allow an even distribution of NMP throughout the membrane and an air drying of its surface. Then, the originally stiff membrane had turned into a soft membrane, which can be applied clinically. (Miguel et al. 2009). Hence, it might be expected

\section{Animals}

Five adult German sheepdogs, weighing more than $20 \mathrm{~kg}$, were used in the present study. The animals were kept in a purpose-designed room for experimental animals and were fed with a standard laboratory diet. The study was evaluated and accepted by the responsible Veterinary Authority (University of Belgrade, Serbia). All surgical procedures including extraction and implant placement were performed under general anaesthesia according to an earlier study (Jung et al. 2007).

\section{Surgical procedure}

Oral prophylaxis comprising of calculus removal and chlorhexidine swabs was performed I week before tooth extraction and I week before the regenerative surgery using drug sedation.

\section{Surgery 1 (tooth extraction)}

From five dogs, the mandibular premolars $\mathrm{P}_{2}$, $\mathrm{P}_{3}, \mathrm{P}_{4}$, and the molar $\mathrm{MI}$ were extracted bilaterally. Wound closure was achieved using resorbable sutures.

\section{Surgery 2 (implantation and lateral ridge} augmentation)

Implantation and regenerative surgery was performed 3 months following tooth extraction. Following mid-crestal incisions of $2.5 \mathrm{~cm}$ in length as well as buccal vertical releasing incisions distal to the canine, a full-thickness flap was carefully elevated. On each side of the mandible, two angular ridge form defects with the following dimensions were created: $9 \mathrm{~mm}$ in the mesio-distal direction, $5 \mathrm{~mm}$ in the apicalcoronal direction, and $7 \mathrm{~mm}$ in the bucco-oral direction. A total of 20 experimental implants with a sandblasted, large-grit, acid-etched surface up to the shoulder of the Straumann Dental Implant System (Straumann AG) with a diameter of $3.3 \mathrm{~mm}$ and a length of $8 \mathrm{~mm}$ were placed obtaining primary stability (Fig. Ia and b).

One implant was placed per defect in such a way that the implant-shoulder was vertically located at the level of the alveolar bone crest at the lingual side. This resulted in a buccal dehiscence defect (Fig. Ib). At this time point, the
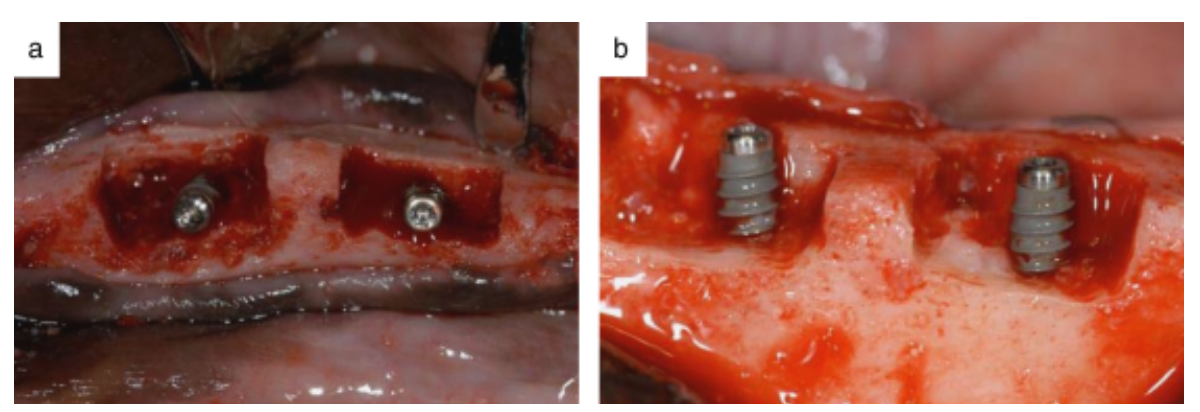

Fig. I. (a and b) Clinical photographs of bony defects before membrane and bone substitute application. The dimensions of the defects were $9 \mathrm{~mm}$ in the mesio-distal direction and $5 \mathrm{~mm}$ in the apical-coronal direction.

vertical defect extension was measured from the top of the implant cylinder shoulder to the first bone-to-implant contact at the buccal aspect.

The four implants inserted in each of the five dogs were randomly allocated to the PLGA/NMP group or RCM group either in combination with DBBM or without resulting in the following four treatment modalities:

Test I - PLGA/NMP membrane only: biodegradable polylactide, polyglycolide, trimethylene carbonate-based and $N$-methylpyrrolidone (NMP)releasing membrane (PLGA/NMP) membrane (Inion ${ }^{\mathbb{B}} \mathrm{GTR}^{\mathrm{TN}}$ ) without bone substitute material.

Test 2 - PLGA/NMP membrane with DBBM: biodegradable PLGA/NMP membrane (Inion $^{\text {R }}$ $\mathrm{GTR}^{\mathrm{TM}}$ ) with DBBM (Bio-Oss ${ }^{\text {(1) }}$ ) as bone substitute material.

Positive control - RCM with DBBM: RCM $\left(\right.$ Bio-Gide $^{\text {[i }}$ ) with DBBM Bio-Oss ${ }^{\mathbb{E}}$ as bone substitute material.

Negative control - RCM only: RCM (BioGide $^{\mathbb{R}}$ ) without bone substitute material.

For the Test 2 and positive control modalities, the DBBM granules were mixed with a sterile saline solution and placed into the bone defects. Before applying the membrane, the horizontal defect extensions were measured from the buccal implant surface to the most buccal aspect of the graft material. Both types of membranes were trimmed and draped over the defect and the adjacent ridge in order to overlap the defect margins 2-3 $\mathrm{mm}$. Each membrane was secured at the buccal aspect with two resorbable trimethylene carbonate/D, L-lactide tacks (Inion Oy) and tucked underneath the lingual flap (Fig. 2a and b). At the test sites, the bioresorbable PLGA/NMP membranes were moistened with sterile saline solution and subsequently the membrane became stiff after approximately $90 \mathrm{~s}$.

To allow a tension-free wound closure, the periosteum of the buccal flap was relieved along the entire base. Thereafter, primary wound closure was obtained with a vertical mattress and interrupted e-PTFE sutures (Gore-Tex ${ }^{\mathbb{B}}$ sutures 5.O, W. L. Gore \& Assoc., Flagstaff, AZ, USA). At the end of each surgical procedure and twice thereafter, analgesic (Baralgin ${ }^{1 B}$ ) was intramuscu-
(C) 2010 John Wiley \& Sons A/S 
larly injected to reduce postoperative pain. The dogs remained on a soft diet for the remainder of the study. The dogs were kept in a purposedesigned cage and allowed once a day $2 \mathrm{~h}$ into an outdoor environment. The sutures were removed io days after surgery.

Three months following regenerative surgery, animals were sacrificed with an overdose of intravenous sodium pentobarbital under deep anaesthesia. All sites were harvested with intact soft tissues.

\section{Histological preparation}

Fixation of the block sections was performed in $4 \%$ formaldehyde for 2 weeks. Following fixation, the specimens were rinsed in running tap water, trimmed and dehydrated in a graded series of increasing ethanol concentrations. Embedding was performed in methylmethacrylate. Tissue blocks were cut into $200-\mu \mathrm{m}$-thick vertical sections using a diamond saw (Exakt, Norderstedt, Germany). The sections were ground and polished to a final thickness of $40-80 \mu \mathrm{m}$ (Exakt), and surface-stained with toluidine blue (Schenk et al. I984).

\section{Histomorphometry}

From each specimen, the central orofacial section through the implant was selected for a quantitative assessment of different linear measurements (vertical and horizontal extension of the regenerated bone), interfaces, and various tissue components by applying standard morphometrical techniques (Weibel et al. I966; Gundersen et al. I988). Measurements were performed directly under a light microscope (Leica Microsystems AG, Heerbrugg, Switzerland).

Linear measurements were performed at a magnification of 40 times in order to calculate the amount of regenerated bone in the former defect area:

(a) The area of regenerated bone was calculated as the area of newly formed bone in $\mathrm{mm}^{2}$ within the area outlined by the membrane.

(b) Bone-to-implant contact was evaluated from the base of the prepared defect to the implant shoulder and was calculated in $\mathrm{mm}$.

(c) The vertical extension of the regenerated bone was measured from the implant shoulder to (i) the highest bone-to-implant contact and (ii) to the highest extension of newly formed bone at the buccal side of the implant. The implant shoulder was set as zero and negative values represent bone tissue below the implant shoulder, whereas positive values reveal bone tissue above the implant shoulder.

(d) To obtain quantitative information about the horizontal extension of regenerated bone, the distance from the outer surface of the regenerated bone to the implant surface
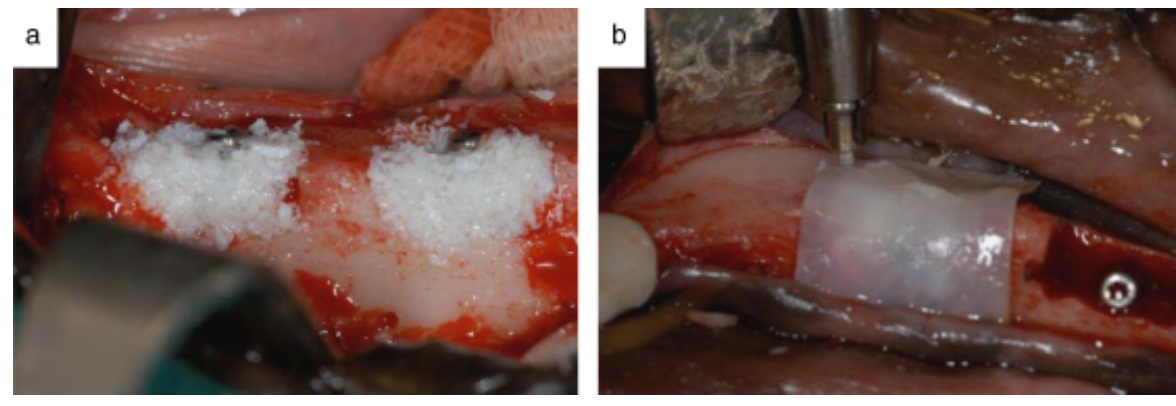

Fig. 2. (a) Clinical photographs of deproteinized bovine bone mineral placed in the bony defects and (b) covered with polylactide/ polyglycolide/ $N$-methyl-2-pyrrolidone membrane held in place by resorbable trimethylene carbonate/D, L-lactide tacks.
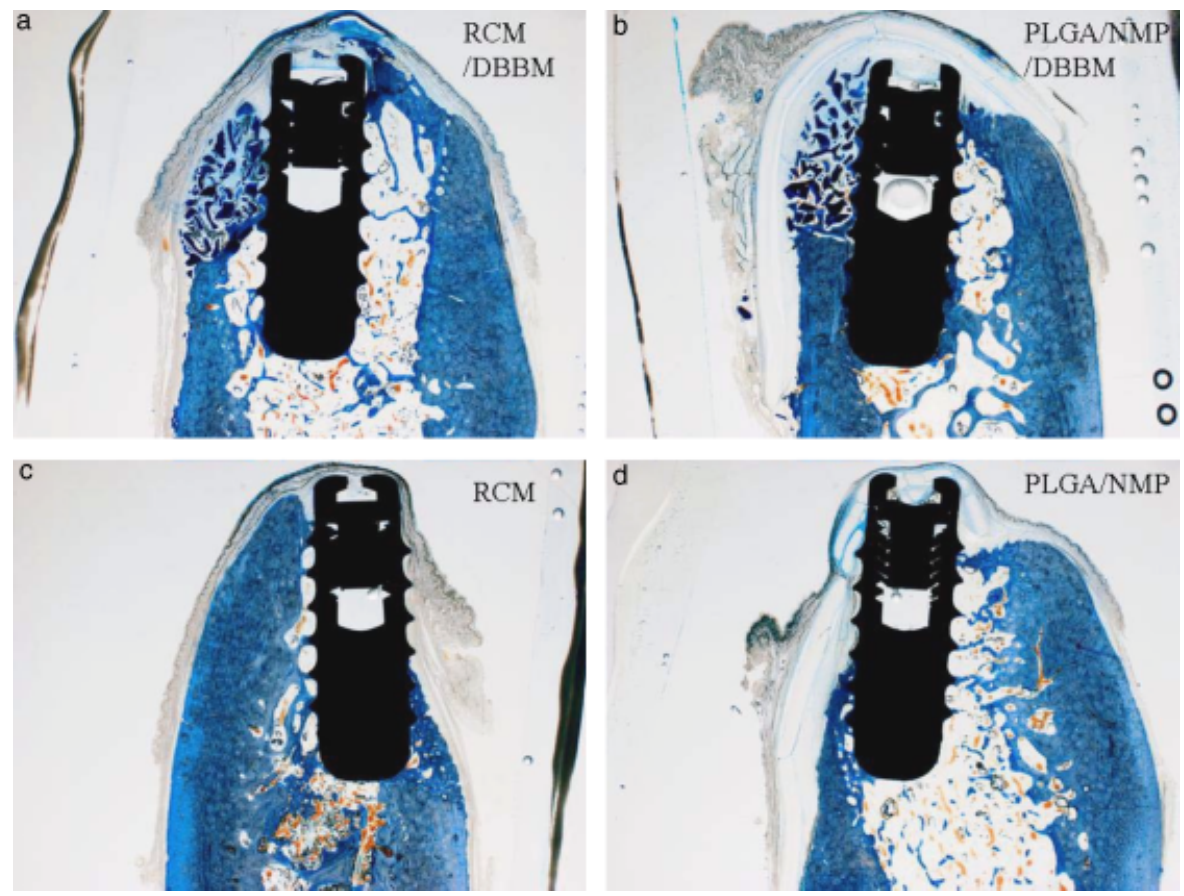

Fig. 3. Histological views of all treatment modalities in a single dog. (a) Resorbable collagen membrane (RCM) with deproteinized bovine bone mineral (DBBM) showing good bone regeneration and (c) without bone substitute material. (b) The polylactide/polyglycolide/ $N$-methyl-2-pyrrolidone (PLGA/NMP) membrane group with bone substitute material (DBBM) shows good bone regeneration even with broken membrane fragments and (d) PLGA/NMP membrane group without bone substitute material showing the remnants of the broken PLGA/NMP membrane.

was measured perpendicular to the implant at the level of the first three threads.

(e) To obtain quantitative information about the horizontal extension of regenerated bone beyond the augmented area, the distance from the outer surface of the regenerated bone to the outer surface of the graft material was measured perpendicular to the implant at the level of the first three threads. Negative values represent graft material exceeding bone tissue horizontally, whereas positive values reveal bone tissue formation beyond the graft material.

\section{Statistical analysis}

All data for statistical variables of new bone formation, membrane material, and non-mineralized tissue were first analysed by a Friedman test and if applicable by a Wilcoxon signed ranks test using the SSPS software version I 8.0 (SPSS Inc., Chicago, IL, USA). The animal was chosen as the unit for statistical analysis. The level of significance chosen in all statistical tests was set at $P<0.05$.

\section{Results}

All surgical procedures went uneventful with the exception of one dog, where residual tooth pieces were found. Because the removal of these pieces led to a very huge defect, no implant could be placed at one of the four defect sites. This situation led to the exclusion of this site from further analysis. Before and after the surgical procedures, all dogs stayed in good health. Soft tissue healing was excellent at all sites and no dehiscence was detected. Histological analysis (Fig. 3d) revealed 
Table 1. Descriptive measurements of the histomorphometric analysis

\begin{tabular}{|c|c|c|c|c|}
\hline & $\begin{array}{l}\text { RCM } \\
\text { DBBM }(N=4)\end{array}$ & $\begin{array}{l}\text { PLGA/NMP } \\
\text { DBBM }(N=5)\end{array}$ & $\mathrm{RCM}(N=5)$ & PLGA/NMP $(N=5)$ \\
\hline Area of bone regeneration $\left(\mathrm{mm}^{2}\right)$ & $9.53 \pm .3 .46$ & $10.03 \pm 5.76$ & $\begin{array}{c}2.53 \pm 2.69 \\
-\quad P<0.032\end{array}$ & $1.27 \pm 1.82$ \\
\hline Bone-to-implant contact $(\mathrm{mm})$ & $3.05 \pm 1.46$ & $2.56 \pm 1.06$ & $\begin{array}{r}1.72 \pm 1.4 \\
-\quad P<0.043\end{array}$ & $0.46 \pm 4.88$ \\
\hline Implant shoulder highest bone implant contact ( $\mathrm{mm})$ & $-1.29 \pm 0.41$ & $-1.39 \pm 0.81$ & $P<0.043$ & $-2.73 \pm 1.14$ \\
\hline Implant shoulder highest bone $(\mathrm{mm})$ & $0.63 \pm 0.91$ & $1.03 \pm 0.61$ & $-2.53 \pm 1.65$ & $-2.63 \pm 1.24$ \\
\hline
\end{tabular}

RCM, resorbable collagen membrane; DBBM, deproteinized bovine bone mineral; PLGA/NMP, polylactide/polyglycolide/N-methyl-2-pyrrolidone.

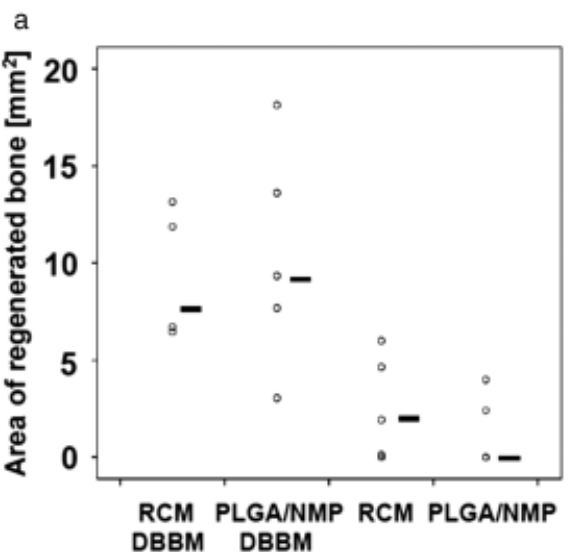

b

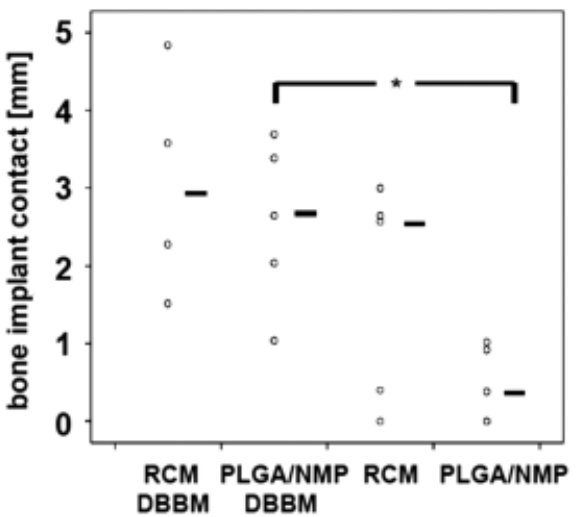

Fig. 4. Analysis of histomorphometry. (a) Area of bone regeneration and (b) bone-to-implant contact. The lines to the right of the values indicate the median of the group. Significant difference between groups is indicated with $\left(^{*}\right)$.

a

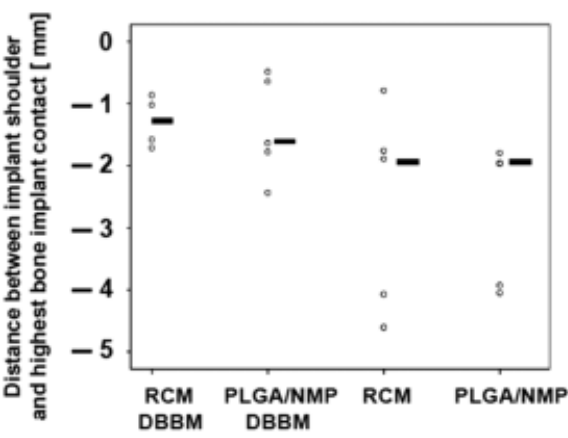

b

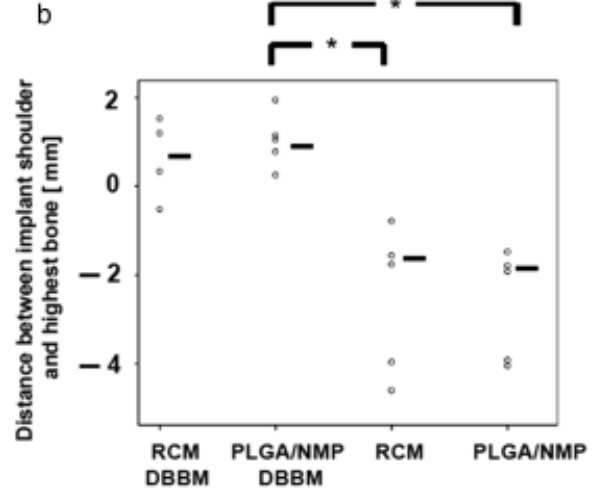

Fig. 5. (a) Distance between implant shoulder and the highest bone-to-implant contact and (b) distance between implant shoulder and the highest extent of bone tissue. The lines to the right of the values indicate the median of the group. Significant differences between groups are indicated with $\left({ }^{*}\right)$.

that in four out of five PLGA/NMP membranetreated defects the membranes had broken into pieces. In the PLGA/NMP membrane/DBBM group, only one membrane had broken (Fig. 3b). Therefore, the tenting effect of the PLGA/NMP membrane is apparently not sufficient to maintain the space in extended defects in dogs. However, when supported by bone substitute material (DBBM), the stability increased substantially. In terms of bone formation, the fracture of the one PLGA/NMP membrane in the PLGA/NMP membrane/DBBM group had no negative impact on bone formation. In contrast to the PLGA/
NMP membrane, all RCMs remained intact at all sites and presumably, maintained their barrier function (Fig. 3a and c).

The histomorphometric data are displayed in Table I. As shown in Fig. 4a, the area of regenerated bone was identical for both membranes in the DBBM group and in the group without DBBM. In addition, the influence of the membrane on the bone/implant interphase was evaluated. As shown in Fig. 4b, the two membranes had no significant influence on the bone-to-implant contact. But without bone substitute material, the overall bone-to-implant contact was higher in the RCM group compared with the PLGA/NMP group. The difference between the PLGA/NMP group with DBBM and without DBBM was significant.

The success of an implant depends on its osseous integration. Therefore, the highest point of bone-to-implant contact and the highest bone tissue level were determined (Fig. 5a and b). The results showed that between the corresponding groups (with and without DBBM) no significant difference could be detected. The difference between the highest bone tissue level of the PLGA/ NMP group with DBBM compared with both membranes without DBBM $(N=5, P=0.034)$ was significant.

Horizontal bone gain was assessed at three different levels (first, second, and third thread). The results revealed no significant difference in horizontal bone formation between the two membranes at any level (Fig. 6a). In addition, we determined bone regeneration beyond the graft material. When compared with RCMs, the PLGA/NMP membrane was capable of inducing bone regeneration horizontally beyond the graft material in the lower region of the defect (Fig. 6b). At the level of the third thread, there was a bigger difference between the two membranes, however, reaching no statistically significant difference.

\section{Discussion}

The present dog study demonstrates that a newly developed PLGA/NMP membrane in combination with a membrane supporting material (DBBM) revealed no significant differences in the majority of the tested parameters compared with the use of an RCM with DBBM. The only small difference of the PLGA/NMP membrane was that on the level of the third thread of the implant, more bone had formed horizontally beyond the graft material in the PLGA/NMP group compared with the RCM group. This small edge might derive from the use of NMP, known to enhance autologous and recombinant bone morphogenetic proteins yielding into enhanced bone regeneration (Miguel et al. 2009). 


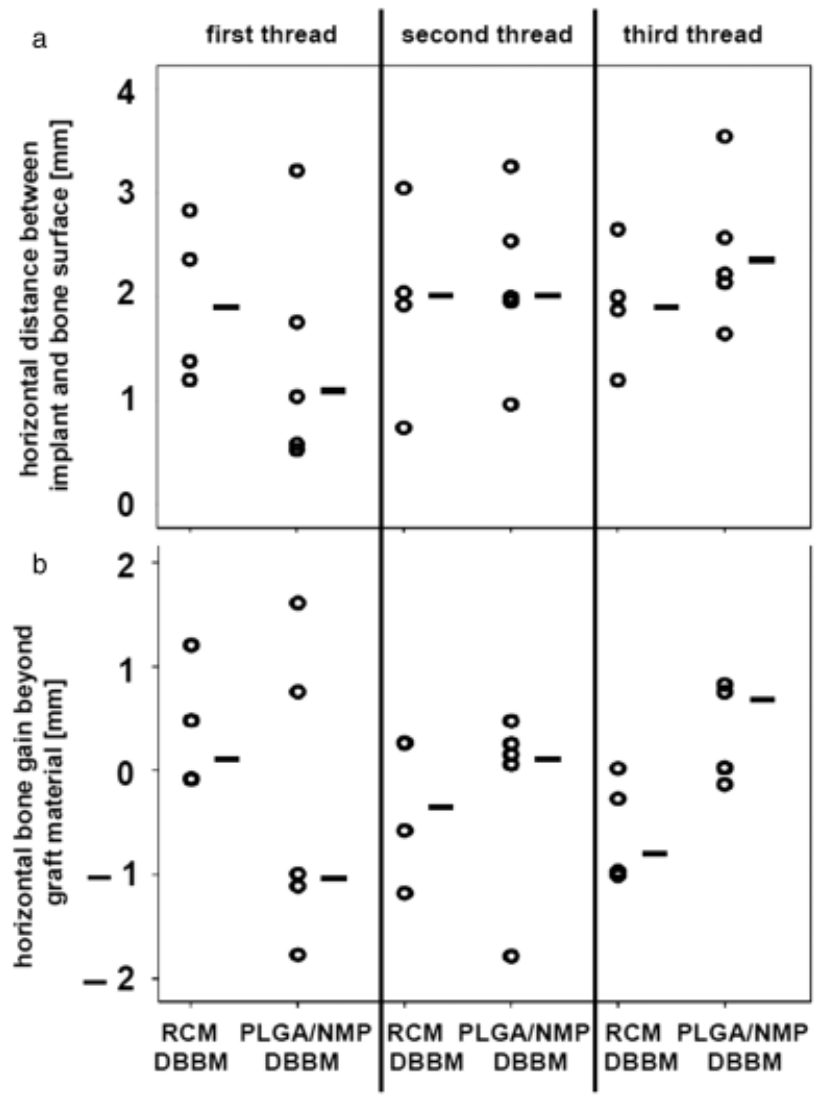

Fig. 6. (a) Horizontal bone gain measured perpendicular to the implant at the level of thread I, 2, and 3. (b) Horizontal bone gain beyond the graft material determined by the distance from the outer surface of the regenerated bone to the outer surface of the graft material measured perpendicular to the implant at the level of thread I, 2, and 3. Positive values indicate that bone had formed horizontally beyond the graft material at the buccal side. No significant differences were observed between the corresponding groups.

The newly developed PLGA/NMP membrane is initially composed of a stiff material (polyglycolide, polylactide). For medical use, the material has to be softened, which is facilitated by incubation in and influx of NMP. Upon placement in the patient and the resulting exposure to body fluids, the softener NMP is released, increasing the stiffness of the membrane again (Pirhonen et al. 2006). The outcome of the present experiment revealed that the regained stiffness is not sufficient to maintain a space for bone ingrowth by its own, because in four of five cases the PLGA/ NMP membrane broke. Therefore, the tenting effect of this membrane on its own is limited. A reason for this outcome might be that the design of the study aimed toward a challenging defect size, which would not be treated without bone substitute material in a human situation. Because the forces applied on the membranes by the dogs could not be controlled beyond the feeding of soft meals, it remains unclear whether a PLGA/NMP membrane can withstand the "normal" forces in humans. In a recent human trial, the same membrane was used to cover extraction sockets of impacted wisdom teeth
(Zwahlen et al. 2009). Histological data and clinical outcome provided no indications for membrane fractures and/or failures. However, one has to take into account that in this human trial the blood clot underneath the membrane had been stabilized by a collagen sponge and that the defects created were four-wall instead of threewall bony defects as in the present dog model.

It is worth mentioning that the failure of the PLGA/NMP membrane led to a worse outcome than the collapsing collagen membrane. This might have two reasons: First, the collagen membrane did not rupture and maintained its barrier function. Therefore, soft tissue in-growth was still prevented in the RCM group, whereas the broken PLGA/ NMP membrane allowed fibrous tissue in-growth into the regenerating area (Fig. $3 \mathrm{~d}$ ). Second, the pieces of the broken PLGA/NMP membrane stacked onto the defect and hindered new bone formation physically, leading to a significant reduction of the bone-to-implant contact (Fig. 4b). When the PLGA/NMP membrane was applied without DBBM compared with its application with DBBM, no significant difference was found between both membranes either applied with or without DBBM.
An earlier study in mongrel dogs (Kohal et al. I999) evaluated the effect of GBR without the application of a bone substitute material around non-submerged implants using different barrier membranes in three groups: (a) an ePTFE membrane, (b) a bioresorbable membrane made from a synthetic copolymer of glycolide and lactide, and (c) no membrane application. The defects $15 \mathrm{~mm}$ apicocoronal $\times 5 \mathrm{~mm}$ mesiodistal) were not filled with a bone substitute material. After a healing period of 6 months, the mineralized bone-to-implant contact in the bioinert e-PTFE membrane group was $5 \mathrm{I} .5 \%$, in the control group $46.3 \%$, and $37.5 \%$ in the biodegradable membrane group. The results of this study indicated that GBR with a bioinert e-PTFE membrane was equal without using bone substitute material. However, compared with the present study, the defect size was much smaller and less challenging, because all four bony walls were preserved.

In combination with the bone substitute material DBBM, RCMs and PLGA/NMP membranes performed equally well in terms of area of bone regeneration, bone-to-implant contact, horizontal bone gain, and all the other parameters tested. One difference was seen in the horizontal extension of bone tissue compared with graft material at the level of the third thread (Fig. 6b). This result might be explained by the fact that NMP released from the membrane increases bone formation more at the bottom of a defect and closer to the membrane. The first vector (closer to the bottom) is most likely due to the higher density of NMP compared with body fluid. The second (closer to the membrane) can be due to the constant release of residual NMP from the membrane. Both not only support the concept of the bioactivity of NMP (Miguel et al. 2009) but also highlight the difficulty in applying it in traditional applications of membranes in humans, because most of the NMP will flow out of the defect site. This in vivo model was able to document the bioactivity of NMP in a calvarial defect model where PLGA/NMP membranes were placed at both sides of the defect. This defect anatomy allowed to trap the released NMP within the bony borders of the bone defect (Miguel et al. 2009). This configuration, in contrast to normal dental applications, might have increased the effective concentration of NMP in the defect and prolonged its exposure to the resident cells close to the defect margins with regenerative potential.

A similar situation in humans concerning the bony walls exists in extraction sockets. Hence, the first clinical trial to evaluate the osteopromotive effect of NMP within the PLGA/NMP membrane was performed in an extraction socket model. The results of that clinical trial with the 
PLGA/NMP membrane showed that old bone density around the defect had increased in the PLGA/NMP group compared with the RCM group (Zwahlen et al. 2009); however, the number of patients and the magnitude of this effect were too low to show a highly significant increase in bone formation or bone density between the groups.

The present study design did not include an empty control in order to assess the amount of spontaneous healing within this type of defects. This would allow to better interpret the outcome of bone regeneration; however, on the other hand,

\section{References}

Cornelini, R., Cangini, F., Martuscelli, G. \& Wennstrom, J. (2004) Deproteinized bovine bone and biodegradable barrier membranes to support healing following immediate placement of transmucosal implants: a short-term controlled clinical trial. International Journal of Periodontics and Restorative Dentistry 24: 555-563.

Gher, M.E., Quintero, G., Assad, D., Monaco, E. \& Richardson, A.C. (I994) Bone grafting and guided bone regeneration for immediate dental implants in humans. Journal of Periodontology 65: 88I-89I.

Gundersen, H.J., Bendtsen, T.F., Korbo, L., Marcussen, N., Moller, A., Nielsen, K., Nyengaard, J.R., Pakkenberg, B., Sorensen, F.B., Vesterby, A. \& West, M.J. (r988) Some new, simple and efficient stereological methods and their use in pathological research and diagnosis. Acta Pathalogica Microbiologica et Immunologica Scandinavica 96: 379-394.

Hammerle, C.H., Jung, R.E., Yaman, D. \& Lang, N.P. (2008) Ridge augmentation by applying bioresorbable membranes and deproteinized bovine bone mineral: a report of twelve consecutive cases. Clinical Oral Implants Research 19: 19-25.

Hammerle, C.H. \& Lang, N.P. (200I) Single stage surgery combining transmucosal implant placement with guided bone regeneration and bioresorbable materials. Clinical Oral Implants Research I2: 9-I8.

Hurzeler, M.B., Kohal, R.J., Naghshbandi, J., Mota, L.F., Conradt, J., Hutmacher, D. \& Caffesse, R.G. (r998) Evaluation of a new bioresorbable barrier to facilitate guided bone regeneration around exposed implant threads. An experimental study in the monkey. International Journal of Oral and Maxillofacial Surgery 27: 31 5-320.

Jung, R.E., Cochran, D.L., Domken, O., Seibl, R., Jones, A.A., Buser, D. \& Hammerle, C.H. (2007) The effect of matrix bound parathyroid hormone on bone regeneration. Clinical Oral Implants Research I8: 319-325.

Jung, R.E., Glauser, R., Scharer, P., Hammerle, C.H., Sailer, H.F. \& Weber, F.E. (2003) Effect of rhBMP-2 the tenting effect of the different membranes could not been assessed.

In conclusion, the newly developed fully synthetic biodegradable PLGA/NMP membrane performs equally as well as the resorbable collagenbased membrane when applied in combination with a bone substitute material. A small increase in the horizontal bone gain beyond the graft material at the level of the third thread of the implant might be attributed to the osteopromotive action of NMP. Without bone substitute material, the PLGA/NMP membrane performed worse than the resorbable collagen-based mem- brane in challenging defects. Therefore, the combination with a bone substitute material is highly recommended.

Acknowledgements: This study was supported from the University of Zürich and the University of Belgrade. A special thanks goes to the histological lab assisted by J. Fierz and A. Tchouboukov. Conflicts of interest: The authors declare no conflicts of interest.

on guided bone regeneration in humans. Clinical Oral Implants Research I4: 556-568.

Kohal, R.J. \& Hurzeler, M.B. (2002) Bioresorbable barrier membranes for guided bone regeneration around dental implants. Schweizer Monatszeitschrift für Zahnmedizin I12: I222-I 229.

Kohal, R.J., Trejo, P.M., Wirsching, C., Hurzeler, M.B. \& Caffesse, R.G. (I999) Comparison of bioabsorbable and bioinert membranes for guided bone regeneration around non-submerged implants. An experimental study in the mongrel dog. Clinical Oral Implants Research Iо: 226-237.

Lang, N.P., Tonetti, M.S., Suvan, J.E., Pierre Bernard, J., Botticelli, D., Fourmousis, I., Hallund, M., Jung, R., Laurell, L., Salvi, G.E., Shafer, D. \& Weber, H.P. (2007) Immediate implant placement with transmucosal healing in areas of aesthetic priority. A multicentre randomized-controlled clinical trial. i. Surgical outcomes. Clinical Oral Implants Research I 8: I 88-I96.

Machtei, E.E. (200I) The effect of membrane exposure on the outcome of regenerative procedures in humans: a meta-analysis. Journal of Periodontology 72: 5 I2-5 I6.

Miguel, B.S., Ghayor, C., Ehrbar, M., Jung, R.E., Zwahlen, R.A., Hortschansky, P., Schmoekel, H.G. \& Weber, F.E. (2009) $N$-methyl pyrrolidone as a potent bone morphogenetic protein enhancer for bone tissue regeneration. Tissue Engineering Part $A$ I5: 2955-2963.

Miller, N., Penaud, J., Foliguet, B., Membre, H., Ambrosini, P. \& Plombas, M. (I996) Resorption rates of 2 commercially available bioresorbable membranes. A histomorphometric study in a rabbit model. Journal of Clinical Periodontology 23: I05 I-I059.

Owens, K.W. \& Yukna, R.A. (200I) Collagen membrane resorption in dogs: a comparative study. Implant Dentistry Io: 49-58.

Parodi, R., Carusi, G., Santarelli, G. \& Nanni, F. (I998) Implant placement in large edentulous ridges expanded by GBR using a bioresorbable collagen membrane. International Journal of Periodontics and Restorative Dentistry I 8: 266-275.
Pirhonen, E.M., Pohjonen, T.H. \& Weber, F.E. (2006) Novel membrane for guided bone regeneration. International Journal of Artificial Organs 29: 834-840.

Rosen, P.S. \& Reynolds, M.A. (200I) Guided bone regeneration for dehiscence and fenestration defects on implants using an absorbable polymer barrier. Journal of Periodontology 72: 250-256.

Schenk, R.K., Olah, A.J. \& Herrmann, W. (I984) Preparation of calcified tissues for light microscopy. In: Dickson, G.R., ed. Methods of Calcified Tissue Preparations, I-56. Amsterdam: Elsevier.

Simion, M., Baldoni, M., Rossi, P. \& Zaffe, D. (I994) A comparative study of the effectiveness of e-PTFE membranes with and without early exposure during the healing period. International Journal of Periodontics and Restorative Dentistry I4: I66-I 80.

von Arx, T., Broggini, N., Jensen, S.S., Bornstein, M.M., Schenk, R.K. \& Buser, D. (2005) Membrane durability and tissue response of different bioresorbable barrier membranes: a histologic study in the rabbit calvarium. The International Journal of Oral (4) Maxillofacial Implants 20: 843-853.

von Arx, T., Cochran, D.L., Schenk, R.K. \& Buser, D. (2002) Evaluation of a prototype trilayer membrane (PTLM) for lateral ridge augmentation: an experimental study in the canine mandible. The International Journal of Oral and Maxillofacial Surgery 3I: I90-I99.

Weibel, E.R., Kistler, G.S. \& Scherle, W.F. (I966) Practical stereological methods for morphometric cytology. Journal of Cell Biology 30: 23-38.

Zhao, S., Pinholt, E.M., Madsen, J.E. \& Donath, K. (2000) Histological evaluation of different biodegradable and non-biodegradable membranes implanted subcutaneously in rats. Journal of Cranio-Maxillofacial Surgery 28: I I6-I 22.

Zwahlen, R.A., Cheung, L.K., Zheng, L.W., Chow, R.L., Li, T., Schuknecht, B., Gratz, K.W. \& Weber, F.E. (2009) Comparison of two resorbable membrane systems in bone regeneration after removal of wisdom teeth: a randomized-controlled clinical pilot study. Clinical Oral Implants Research 20: I084-I091. 